\title{
Enurese Noturna na Adolescência: Tratamento em Grupo e Individual
}

\author{
Noel José Dias da Costa \\ Edwiges Ferreira de Mattos Silvares \\ Universidade de São Paulo
}

\begin{abstract}
RESUMO
Objetivou-se comparar os resultados de um programa de intervenção comportamental para enurese noturna, tendo como recurso terapêutico o aparelho nacional de alarme de urina, aplicado, em duas clinica-escolas brasileiras, em adolescentes, em grupo e individualmente. Os participantes foram adolescentes e seus responsáveis, pela demanda de participação ativa da família. Os participantes $(\mathrm{N}=22)$ foram comportamentalmente orientados. Do total dos participantes 14 foram atendidos em dois grupos ( $\mathrm{G} 1, \mathrm{~N}=8$; $\mathrm{G} 2, \mathrm{~N}=6$ ) e oito foram atendidos individualmente (G3). O critério de sucesso foi duas semanas consecutivas sem episódios enuréticos (“molhadas") e o critério de alta, oito semanas consecutivas sem molhadas. A duração máxima do atendimento foi de 40 semanas. Utilizouse o registro simples de "molhadas", aplicado ao longo de todo atendimento. Dos participantes do atendimento coletivo, 11 (78,6\%) alcançaram sucesso e nove (64,2\%) alcançaram alta. No atendimento individual, sete $(87,5 \%)$ alcançaram o sucesso, e seis (75\%) alcançaram alta. Essa diferença entre grupos não alcançou significância estatística. Entretanto, o atendimento individual demonstrou ser mais eficiente que o coletivo em promover a alta, quando ambos foram comparados no intervalo de seis e dez meses, ( $p=0,030$ e 0,045 , respectivamente), um resultado significante do ponto de vista estatístico. Nos demais aspectos, os dois tipos de atendimento apresentaram resultados comparáveis. Independente do tipo de atendimento, foi encontrada uma associação entre faltas e pior desempenho no tratamento. Conclui-se que o tratamento em grupo para enurese noturna na adolescência é viável, especialmente considerando-se a relação custo/benefício.
\end{abstract}

Palavras-chave: aparelho de alarme; enurese; adolescência; atendimento individual; atendimento em grupo.

\section{ABSTRACT \\ Nocturnal Enuresis in Adolescence: Individual and Group Treatment}

The results of a behavioral intervention program for nocturnal enuresis were compared using a national urine alarm device, applied in groups and individually, to adolescents from two Brazilian University clinics. The participants included adolescents and their parents in a program of active family participation. The participants $(\mathrm{N}=22)$ were behaviorally guided. Some participants were assisted in group (total $\mathrm{N}=14 ; \mathrm{G} 1, \mathrm{~N}=8 ; \mathrm{G} 2, \mathrm{~N}=6$ ) while eight were assisted individually (G3). Treatment success was determined following two consecutive weeks without "wet episodes" and the criterion for discharge was eight consecutive weeks without any wet episodes. The maximum length of the assistance was 40 weeks. Results in the current report focus on the analyses of the simple "wet episode" recordings, used throughout the treatment. From the assisted group, 11 participants (78.6\%) succeeded and nine (64.2\%) were discharged. Of those undergoing individual assistance, seven (87.5\%) succeeded, and six (75\%) were discharged. These differences did not reach statistical significance. The individual assistance group, within the interval of six and ten months, proved to be more efficient in promoting discharge than the group assistance service, $(p=0,030$ and 0,045 , respectively). In the other aspects, the two types of assistance demonstrated comparable results. Missing therapeutic sessions were associated with poorer treatment performance, independently of assistance type. It is concluded that group treatment for nocturnal enuresis is feasible in university clinics, especially considering the cost/benefit relationship.

Keywords: urine alarm device; enuresis; adolescence; individual assistance; group assistance. 
A enurese é uma desordem funcional de eliminação (Mellon \& Houts, 1995) caracterizada pelo urinar acidental ou incontrolado na ausência de patologia urológica ou neurológica (Doleys, 1977). É considerada um problema biocomportamental, no qual interagem fatores orgânicos e psíquicos. O Manual Diagnóstico e Estatístico de Transtornos Mentais (DSM-IV) estabelece quatro critérios para diagnóstico da enurese:

a) micção repetida, diurna ou noturna, na cama ou na roupa; b) a micção deve ocorrer no mínimo duas vezes por semana por pelo menos três meses, ou então causar um sofrimento ou prejuízo significativo no funcionamento social, acadêmico (ocupacional) ou outras áreas importantes na vida do indivíduo; c) é esperado que a continência ocorra com a idade cronológica de no mínimo cinco anos, ou para crianças com atrasos de desenvolvimento, idade mental de no mínimo cinco anos; d) a incontinência urinária não se deva exclusivamente aos efeitos fisiológicos diretos de uma substância (por exemplo, diuréticos) ou a uma condição geral (por exemplo, diabete, espinha bífida, transtorno convulsivo) (American Psychiatric Association, 2003, p.106).

Djurhuus e Rittig (2002) afirmam que uma das causas da enurese noturna é a deficiência na secreção do hormônio antidiurético vasopressina (ADH), mas que outros mecanismos atuam concomitantemente no organismo como a capacidade de armazenamento da bexiga e a atividade dos músculos detrussores. As hipóteses mais favorecidas são aquelas que focalizam deficiências na secreção noturna do ADH e nas respostas musculares necessárias para inibir a micção.

A enurese pode desencadear problemas emocionais para o adolescente como ansiedade, vergonha, tristeza, desânimo, auto-estima baixa e isolamento social (Butler, 2004; Butler, Holland e Robinson, 2001). Além disso, pode também afetar os pais, ocasionando sentimento de culpa, vergonha, angústia e ansiedade (Costa \& Silvares, 2003). Apesar de a maioria dos pais serem suportivos, cerca de $30 \%$, tornam-se intolerantes à enurese noturna de seus filhos (Butler, 1994). Principalmente para esses, a enurese pode ter um efeito marcante sobre a dinâmica familiar.

A enurese pode tornar-se um problema de grande magnitude em razão de vários motivos. Primeiro, porque desenvolve o sentimento de incompetência, depois porque gera mal estar no ambiente doméstico, pela aversão que provoca nas outras pessoas, que geralmente respondem com hostilidade ou discriminação. Por isso, é imperativa a implementação de méto- dos de tratamento dessa queixa que atinge cerca de 3\% dos adolescentes (Butler e cols, 2001).

Os tratamentos existentes para enurese vão desde o uso de agentes farmacológicos, como antidepressivos tricíclicos, desmopressina e anticolinérgicos, até a terapia comportamental, que inclui técnicas como treinamento de retenção de urina, treinamento de contenção, uso de aparelho de alarme e terapia motivacional (Moffat, 1997; Van Kampen e cols, 2002). As pesquisas sobre os tratamentos com o alarme de urina, nos últimos 60 anos, evidenciam a superioridade deste método sobre todos os outros atualmente disponíveis, tanto no que se refere à superação dos episódios enuréticos quanto às baixas ocorrências de recaídas, que no caso dos tratamentos farmacológicos chegam a $70 \%$, ao passo que no uso do alarme chegam a 35\% (Hirasing, Van Leerdam, Bolk-Bennink \& Koot, 2002; Houts, 1991; Houts, Berman e Abramson, 1994; Silva, 2004; Van Kampen e cols, 2002).

O aparelho de alarme utilizado nesse estudo é o de cabeceira (tipo bell-and-pad), que tem uma unidade de alarme, para ser colocada ao lado da cama e ligada por um cabo a uma unidade sensora (tapete-sensor flexível de poliéster), que é colocada sob o lençol. Ao contato com a urina, o sistema aciona o alarme. A criança então acorda, desliga o aparelho e vai ao sanitário para terminar de urinar. Depois, com a ajuda dos pais, troca a roupa e o lençol molhados, enxuga o tapete-sensor, liga o aparelho novamente e volta a dormir. Dessa forma, o organismo da criança é condicionado a produzir as mudanças necessárias ao controle urinário.

Como mencionado, a participação dos pais ou cuidadores é fundamental nesse tratamento. Por isso é indicado o modelo triádico de atendimento comportamental, que, segundo Silvares (1995), possibilita a utilização de tipos de intervenções adequados às especifidades das diferentes famílias. Esse modelo supõe várias formas de intervenção, sendo as mais comuns: a) Treino de pais em manejo de contingências (Hawkins, Peterson, Shweid \& Bijou, 1966); b) Contrato de Contingências (Miller \& Kelley, 1994) e c) o Treino Familiar em Resolução de Problemas (Tharp \& Wetzel, 1969), a forma utilizada no presente estudo.

No Brasil, a utilização desse tratamento é ainda incipiente, devido à escassez de pesquisa, de informação e de recursos. Ainda assim, desde 1992, o Projeto Enurese, do Instituto de Psicologia da Universidade de São Paulo, vem atendendo crianças e adolescentes a fim de pesquisar formas de implementá-lo no contexto 
do Brasil. O trabalho no Projeto Enurese está inserido na atuação da clínica-escola de psicologia, que tem como objetivos o ensino, a pesquisa e o atendimento de um contingente da população com alta demanda por serviços de saúde mental. Essa população, usuária dos serviços de saúde pública, é estimada em 140 milhões de pessoas subtraindo-se as pessoas atendidas pelo setor de medicina supletiva - medicina de grupo, cooperativas médicas, seguradoras do ramo saúde e planos auto-administrados (Coelho, 2007).

Uma das formas de diminuir os custos de saúde é a utilização do atendimento em grupo, uma prática já consolidada para o tratamento de várias queixas, tanto em hospitais-escola como na rede pública de saúde (Kaplan \& Sadock, 1996). Há poucas pesquisas sobre a eficácia do atendimento de adolescentes enuréticos no formato em grupo ou individual com uso de aparelho de alarme. Nesses estudos, segundo Houts (2003) os resultados obtidos foram comparáveis, com exceção de diferentes taxas de recaída em decorrência de diferentes formas de superaprendizagem, que consiste na ingestão gradual de água por parte do adolescente antes de dormir, como prevenção de recaída. Em seu estudo, García, Veja e Pagés (2002), apresentam os seguintes benefícios para o atendimento em grupo: a) constitui-se um reforçador importante frente à falta de motivação dos adolescentes e seus familiares; b) torna a sessão terapêutica mais agradável, pela diversidade das atividades que possibilita realizar; c) proporciona um ambiente saudável e seguro para a exposição de um problema guardado em segredo pela família, o que permite uma troca de experiências, diminuindo a aversividade do fato.

\section{Objetivo}

O objetivo deste foi investigar a eficiência da intervenção comportamental em grupo com uso de aparelho de alarme para adolescentes com enurese, comparada com o atendimento individual realizados em clinicas-escola.

\section{Método}

Este trabalho foi realizado de 2001 a 2004 na Clínica-escola do Instituto de Psicologia da Universidade de São Paulo - USP e no Centro de Atendimento e Apoio ao Adolescente - CAAA, da Universidade Federal de São Paulo - Unifesp.

Participaram desse estudo, 22 adolescentes, entre 11 e 17 anos, com queixa de enurese noturna distribuídos em três grupos como demonstrado na Tabela 1 .

TABELA 1

Distribuição dos Participantes por Grupos, Gênero, Idade Média e Local de Atendimento

\begin{tabular}{llccc}
\hline Grupo/atendimento & Local & Feminino & Masculino & *Idade (média) \\
\hline G1/coletivo & Clínica-escola IP/USP & 4 & 4 & 11,3 \\
G2/coletivo & Clínica-escola IP/USP & 3 & 3 & 12,0 \\
G3/individual & CAAA - Unifesp & 2 & 6 & 12,8 \\
\hline Total & & 9 & 13 & 12,0
\end{tabular}

Os grupos foram formados de forma randômica; considerando-se também a proximidade da residência dos clientes com o centro de pesquisa. Após aprovação do projeto de pesquisa pelos Comitês de Ética em Pesquisa da Faculdade de Saúde Pública da Universidade de São Paulo - USP e do Departamento de Pediatria da Universidade Federal de São Paulo - Unifesp, foi realizada divulgação do projeto na comunidade, através de cartazes, anúncio em revista de saúde e pela Agência Usp, da Escola de Comunicação e Artes - ECA.

Foram excluídos do estudo participantes que apresentassem as seguintes condições: a) idade inferior a
10 anos e meio ou maior do que 19 anos; b) que estivessem, no momento da procura por nosso atendimento, em outro atendimento para EN; c) que tivessem enurese diurna; d) que tivessem distúrbios de ordem renal ou neurológica; e) que apresentassem infecção crônica do trato urinário.

\section{Instrumentos}

Foram utilizados os seguintes instrumentos, respondidos individualmente: a) Inventário de Comportamentos da Infância e Adolescência - CBCL (Child Behavior Checklist) (Achenbach, 1991); b) Escala de 
Intolerância - EI (Morgan \& Young, 1975); c) Formulário de Avaliação de Enurese - FAE (Blackwell, 1989); Youth Self Report - YSR - versão do CBCL para ser respondida pelo próprio adolescente; e) Registro simples de descontrole enurético ao longo do atendimento. $\mathrm{O}$ presente trabalho se restringiu à análise dos resultados deste último recurso de avaliação em função de seus objetivos e limite de espaço. Foi utilizado também um Termo de Consentimento que, depois de lido e esclarecido aos pais, foi assinado por eles.

\section{Procedimento}

O atendimento se deu através de sessão semanal de aproximadamente 40 minutos, para os adolescentes, seguida de uma sessão de aproximadamente $30 \mathrm{mi}$ nutos para seus pais. Na sessão com os adolescentes foram tratadas questões relativas à enurese e seu tratamento e outros temas relativos a ela, como relacionamento familiar, punição dos pais, limitações sociais etc. Nas sessões com os pais realizou-se intervenção psicoeducacional, oferecendo informações sobre a queixa e seu tratamento, sobre o papel dos cuidadores e as atitudes mais adequadas frente ao problema. Eventualmente, dedicou-se um período após a reunião do grupo para um breve atendimento individual, quando surgia a necessidade. Durante o atendimento aos pais, os adolescentes participavam de atividades lúdicas monitoradas por uma bolsista de iniciação científica, exceto os atendidos individualmente, que aguardavam pelos pais em sala de espera.

Estabeleceu-se o prazo máximo de 40 semanas para o atendimento de cada grupo. Esta decisão resultou de análise efetuada pela equipe do Projeto Enurese, com base na experiência do atendimento a outros grupos com a mesma queixa.

\section{Forma de Análise de Dados}

De acordo com a literatura (Butler, 1994; Houts, 1991; Jensen \& Kristensen, 2001) permanecer sem episódio de molhadas, acidente enurético, durante 14 dias (duas semanas) consecutivos é característica de sucesso inicial no tratamento para EN. Neste estudo, este critério foi adotado para discriminar os casos bem sucedidos e menos bem sucedidos em relação ao tratamento para enurese. O critério de alta foi o de permanecer oito semanas consecutivas sem apresentar episódio de molhadas. Para análise estatística foi utilizado o Teste de Igualdade da Distribuição de Sobrevida (Log Rank).

\section{Resultados e Discussão}

Os resultados demonstraram que o critério de sucesso foi alcançado por $81,8 \%$ do total dos participantes ao término da intervenção. No atendimento coletivo, foi obtido por $78,6 \%$ dos participantes, e no atendimento individual esse índice foi de $87,5 \%$. Não houve diferença entre os resultados dos dois tipos de atendimento do ponto de vista estatístico $(\mathrm{p}=0,148)$.

Os resultados demonstraram ainda não haver diferença significativa entre a taxa semanal média de episódios dos participantes do atendimento coletivo e a dos participantes do atendimento individual $(\mathrm{p}=0,283)$, antes do tratamento. O mesmo não ocorreu também quanto à semana de sucesso $(\mathrm{p}=0,989)$; à semana de alta $(p=0,270)$; à incidência mensal de sucesso $(\mathrm{p}=0,652)$ e à incidência mensal de alta $(\mathrm{p}=0,317)$, conforme demonstrado na Tabela 2.

TABELA 2

Freqüência Semanal de Episódios de Molhadas, Semana de Sucesso, Incidência Mensal, Semana de Alta Incidência Mensal, Risco de Sucesso e de Alta Média Inicial, Semana de Sucesso, em Função do Tipo

\begin{tabular}{|c|c|c|c|c|c|c|c|}
\hline $\begin{array}{c}\text { Tipo de } \\
\text { atendimento }\end{array}$ & $\begin{array}{l}\text { Freqüência } \\
\text { semanal média } \\
\text { (dp) }\end{array}$ & $\begin{array}{l}\text { Semana de } \\
\text { sucesso média } \\
\text { (dp) }\end{array}$ & $\begin{array}{c}\text { Incidência } \\
\text { mensal de } \\
\text { sucesso (\%) }\end{array}$ & $\begin{array}{c}\text { Semana de alta } \\
\text { média (dp) }\end{array}$ & $\begin{array}{c}\text { Incidência } \\
\text { mensal de } \\
\text { alta (\%) }\end{array}$ & $\begin{array}{c}{ }^{*} \text { Risco de } \\
\text { sucesso } \\
(\%)\end{array}$ & $\begin{array}{c}{ }^{*} \text { Risco de } \\
\text { alta (\%) }\end{array}$ \\
\hline Coletivo & $5,6(1,65)$ & $15,9(5,24)$ & 14,9 & $28,7(8,77)$ & 6,3 & 71,4 & 28,6 \\
\hline Individual & $4,8(2,12)$ & $15,9(11,2)$ & 18,5 & $23,5(8,07)$ & 10,9 & 75,0 & 62,5 \\
\hline Valor $p$ & 0,283 & 0,989 & 0,652 & 0,270 & 0,317 & 0,212 & 0,030 \\
\hline
\end{tabular}

Nota. Considerando o início da coorte como primeira sessão.

*Utilizando intervalo de tempo de 0 a 6 meses 
Os resultados do Teste de Igualdade de Sobrevida demonstraram que a probabilidade (risco) de um participante do atendimento coletivo alcançar alta no tratamento em intervalo de tempo de 0 a 6 meses é de $28,6 \%$, e no atendimento individual a probabilidade é de $62,5 \%$. Com o intervalo de tempo de 0 a 10 meses, a probabilidade é de $51,7 \%$ no atendimento coletivo, e de $81,3 \%$ no atendimento individual, sendo essas diferenças significativas do ponto de vista estatístico ( $p=0,030$ e 0,045 , respectivamente). Para estes intervalos de tempo, o atendimento individual demonstrouse superior ao coletivo.
Verificou-se ainda que os participantes com mais de $20 \%$ de faltas (oito) utilizaram 6,8 vezes mais tempo para atingir o critério de sucesso do que aqueles com menos de $10 \%$ de faltas (quatro) independentemente do tipo de tratamento. Esta diferença é significativa do ponto de vista estatístico $(p=0,004)$. Este dado confirma aqueles apresentados por Silva (2004), que atendeu grupos de crianças com essa mesma queixa e verificou que os participantes com menor número de faltas alcançaram sucesso com menor número de sessões.

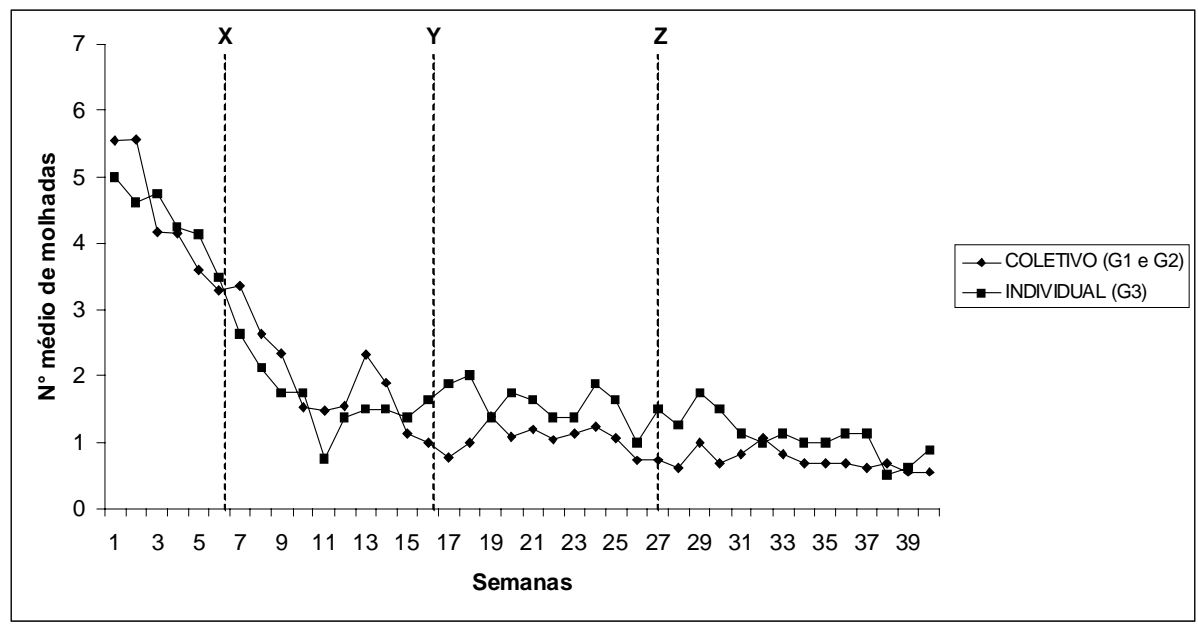

Figura 1. Perfil do número médio de molhadas por tipo de atendimento ( $X=$ Início do uso do aparelho de alarme; $Y$ = Semana de sucesso; $Z$ = Semana de alta).

Através de análise da evolução conjunta dos dois tipos de atendimentos pela Figura 1 é possível constatar a grande semelhança existente entre eles na resposta ao tratamento. Ambos demonstraram-se eficientes em diminuir a freqüência de episódios, havendo apenas discreta diferença no resultado final, que apresenta os dois com a média de episódios muito próximas (coletivo $=0,5$ e individual $=0,8$ ), sendo ambas abaixo de uma molhada semanal. Entretanto, após a semana de sucesso, o atendimento coletivo tendeu sistematicamente a apresentar uma taxa de molhadas inferior ao atendimento individual. Para os dois formatos de atendimento houve diminuição da taxa média de molhadas antes mesmo do início do uso do aparelho, o que parece indicar a importância das sessões terapêuticas.

\section{CONCLUSÃO}

Este estudo mostrou a validade do modelo de tratamento em grupo para adolescentes com enurese noturna, confirmando sua eficiência. A diminuição da freqüência de molhadas seguiu tendência semelhante nos grupos de ambas as modalidades de atendimento. Entretanto, o estudo demonstrou que através do atendimento individual há maior probabilidade de atingir o critério de sucesso e de alta do que através de o coletivo, dentro do mesmo período de tempo. Além disso, este estudo evidenciou que o tratamento em grupo é uma importante alternativa para as unidades públicas de saúde, especialmente se considerar a relação custo-benefício. $\mathrm{O}$ atendimento grupal possibilita atender um contingente maior de clientes num mesmo horário e com um mesmo profissional tornando-se uma solução necessária e vantajosa, tendo como efeito a redução dos custos dos serviços de saúde e das filas de espera.

Por fim, o estudo evidenciou a necessidade da pesquisa sistemática sobre esse tipo de atendimento, tanto para responder questões como as que nortearam o presente estudo quanto para compreender as variáveis envolvidas nos casos menos bem sucedidos. A importância desta busca torna-se evidente se considerarmos que o adolescente enurético, se não tratado, será um adulto enurético em potencial. 


\section{REFERÊNCIAS}

Achenbach, T. M. (1991). Manual for the child behavior checklist/418 and 1991 profile. Burlington: University of Vermont.

American Psychiatric Association (2002). Manual diagnóstico e estatístico de transtornos mentais ( $4^{\mathrm{a}}$ ed.) (C. Dornelles, Trad.). Porto Alegre: Artmed.

Blackwell, C. (1989) A guide to enuresis - A guide to a treatment of enuresis for professionals. Bristol: Enuresis Resource and Information Center.

Butler, R. J. (2004). Childhood nocturnal enuresis: Developing a conceptual framework. Clinical Psychological Review, 24, 909-931.

Butler, R. J. (1994). Nocturnal Enuresis: The child's experience. Oxford: Butterworth-Heinemann.

Butler, R.J., Holland, P., \& Robinson, J. (2001) Examination of the structured withdrawal program to prevent relapse of nocturnal enuresis. Journal of Urology, 166, 2463-2466.

Coelho, I. B. (2007). Os impasses do SUS. Ciência \& Saúde Coletiva, 12(2), 1413-8123.

Costa, N. J. D., \& Silvares, E. F. M.(2003). Enurese na adolescência: Estudo de caso com intervenção comportamental. Interação em Psicologia, 7(1), 9-18.

Djurhuus, J. C., \& Ritting, S. (2002) Nocturnal enuresis. Current Opinion in Urology, 12, 317-320.

Doleys, D. M. (1977). Behavioral treatments for nocturnal enuresis in children: A review of the recent literature. Psychological Bulletin, 84, 30-54.

García, F., Vega, E, Pagés, P. (2002). Psicoterapia de grupo para la enuresis nocturnal: Processos y resultados. Revista Argentina de Clínica Psicológica, 11, 35-47.

Hawkins, R. F., Peterson, R. F., Schweid, E., \& Bijou, S. W. (1966). Behavior therapy in the home: Amelioration of problem parent-child with the parent in a therapeutic role. Journal of Experimental Child Psychology, 4, 99-107.

HiraSing, R. A., van Leerdam, F. J. M., Bolk-Bennink, L. F., \& Koot, H. M. (2002) Effect of dry bed training on behavioral problems in enuretic children. Acta Paedriatrica, 91, 960-964.

Houts, A. C. (2003). Behavioral treatment for enuresis. In A. E. Kazdin \& J. R.Weisz (Orgs.), Evidence-based psychotherapies for children and adolescents (pp. 389-406). New York: The Guilford Press.

Houts, A. C., Berman, J. S., \& Abramson, H. (1994). Effectiveness of psychological and pharmacological treatments for nocturnal enuresis. Journal of Consulting and Clinical Psychology, 62, 737-745.

Houts, A. C. (1991). Nocturnal enuresis as a biobehavioral problem. Behavior Therapy, 22, 33-151.

Jensen, N., \& Kristensen, G. (2001). Frequency of nightly wetting and the efficiency of alarm treatment of nocturnal enuresis. Scandinavian Journal of Urology and Nephrology, 35, 357-363.

Melo, M. H. S. (2003). Crianças com dificuldades de interação no contexto escolar: Uma intervenção multifocal. Tese de doutorado não-publicada, Universidade de São Paulo.

Mellon, M. W., \& Houts, A. C. (1995). Eliminations disorders. Em M. Cimmerman \& M. Hersen (Orgs.), Handbook of child behavior therapy in the psychiatric setting (pp. 341-346). New York: Guilford Press.

Miller, D. L., \& Kelley, M. L. (1994). The use of goal setting and contingency contracting for improving children's homework performance. Journal of Applied Behavior Analysis, 27(1), 73-83.

Moffat, M. E. K. (1997). Nocturnal enuresis: A review of the efficacy of treatments and practical advice for clinicians. Journal of Developmental and Behavioral Pediatrics, 18, 49-56.

Morgan, R. T. T., \& Young, G. C. (1975). Parental attitudes and the conditioning treatment of childhood enuresis. Behavior Research and Therapy, 13, 197-199.

Oliveira, D. S., Santos, G. T., \& Silvares, E. F. M.(2000). A enurese infantil e o uso de alarme no seu controle. Em E. F. M. Silvares (Org.), Estudos de caso em psicologia clínica comportamental infantil, (pp. 121-156). Campinas: Papirus.

Silva, P. L. B. (2003). Serviços de saúde: O dilema do SUS na nova década. São Paulo Perspectiva, 17(1), 69-85.

Silva, R. A. P. (2004). Enurese noturna: Grupo de terapia infantil associado a aparelho de alarme e grupo de orientação a pais. Dissertação de mestrado não-publicada, Universidade de São Paulo.

Silvares, E. F. M. (1995) Modelo triádico no contexto de terapia comportamental com famílias. Psicologia: Teoria e Pesquisa, 11, 235-41.

Tharp, R. G., \& Wetzel, R. J. (1969). Behavior modification in the natural environment. New York: Brunner/Mazel.

Van Kampen, M., Bogaert, G., Feys, H., Baert, L., De Raeymaeker, I., \& De Weerdt, W. (2002). High inicial efficacy of fullspectrum therapy for nocturnal enuresis in children and adolescents. British Journal of Urology, 90, 84-87.

Recebido: 16/01/2007

Última revisão: 04/12/2007

Aceite final: 10/12/2007

\section{Sobre os autores:}

Noel José Dias da Costa: Mestre e doutorando do Departamento de Psicologia Clínica do Instituto de Psicologia da Universidade de São Paulo.

Edwiges Ferreira de Mattos Silvares: Professora titular do Departamento de Psicologia Clínica do Instituto de Psicologia da Universidade de São Paulo.

Endereço para correspondência: Edwiges Ferreira de Mattos Silvares - Av. Prof. Mello Moraes, 1.723 - Cidade Universitária - São Paulo - SP. Endereço eletrônico: efdmsilv@usp.br 